Western University

Scholarship@Western

Management and Organizational Studies

Management and Organizational Studies

Publications

Department

$4-2018$

\title{
When Public Recognition for Charitable Giving Backfires: The Role of Independent Self-Construal
}

\author{
Bonnie Simpson \\ Katherine White \\ Juliano Laran
}

Follow this and additional works at: https://ir.lib.uwo.ca/mospub

Part of the Business Administration, Management, and Operations Commons, and the Work, Economy and Organizations Commons 
When Public Recognition for Charitable Giving Backfires:

The Role of Independent Self-Construal

(In press, Journal of Consumer Research)

BONNIE SIMPSON

KATHERINE WHITE

JULIANO LARAN

Bonnie Simpson (bonnie.simpson@uwo.ca) is Assistant Professor of Consumer Behavior, DAN Management and Organizational Studies, Western University, London, Ontario, Canada, N6A 5C2. Katherine White (katherine.white@sauder.ubc.ca) is Professor of Marketing and Behavioural Science, Sauder School of Business, University of British Columbia, Vancouver, BC, Canada, V6T 1Z2. Juliano Laran (laran@miami.edu) is Professor of Marketing, University of Miami, Coral Gables, FL, 33124-6524. Correspondence: Bonnie Simpson. The authors thank Joey Hoegg for her helpful comments on an earlier draft of this manuscript, and the editorial and review team for their support. Supplementary materials are included the web appendix accompanying the online version of this article. 


\begin{abstract}
This research examines the effectiveness of public recognition in encouraging charitable giving, demonstrating that public recognition can sometimes decrease donations. While previous work has largely shown that making donations visible to others can motivate donors, the present research shows that the effectiveness of public recognition depends on whether potential donors are under an independent (i.e., separate from others) or interdependent (i.e., connected with others) self-construal. Across seven experimental studies, an independent self-construal decreases donation intentions and amounts when the donor will receive public recognition compared to when the donation will remain private. This effect is driven by the activation of an agentic motive, wherein independents are motivated to make decisions that are guided by their own goals and self-interests, rather than being influenced by the opinions and expectations of others. This research contributes to the understanding of the nuanced roles of both public recognition and self-construal in predicting donation behavior.
\end{abstract}

Keywords: self-construal; charitable donations; public recognition; agency 
Individual donors represent the largest source of charitable giving in the United States, totaling $71 \%$ of aggregate giving (Giving USA Foundation 2016). In fact, donations by individual Americans increased 3.8\% in 2015 over 2014, to \$268.58 billion. While this indicates a positive trend, a less encouraging sign is the fact that Americans are not increasing their charitable giving relative to their disposable income, especially at a time when fundraisers need it more (Giving USA Foundation 2016). Charitable organizations and researchers are thus very interested in increasing the effectiveness of appeals made to individual donors (Kristofferson, White, and Peloza 2014; Lee, Winterich, and Ross 2014; Winterich and Zhang 2014).

One tactic commonly used by charitable organizations is to publicly recognize acts of giving by individuals, soliciting for donations in ways that make prosocial actions observable by others (Andreoni and Petrie 2004; Harbaugh 1998). Past research supports the effectiveness of this strategy, suggesting that people are more likely to donate and donate more when they are given public recognition for their philanthropic acts (Benabou and Tirole 2006; Harbaugh 1998; Karlan and McConnell 2014). This recognition may be formal, such as displaying the names of donors on the wall of a building (Harbaugh 1998), displaying the names on a decal (Children's Miracle Network 2016), printing the names in a newsletter or magazine (Basil et al. 2009; Kotler and Lee 2005), or publishing donor information on a website (Winterich et al. 2013; Kristofferson et al. 2014). It may also be informal, such as when the context allows for the donor's behavior to be publicly observable by others (White and Peloza 2009).

While the operationalization of public recognition varies widely in literature and practice, consistent across different forms of public recognition is the fact that the donor perceives that others know their decision to donate or not will be made public. We thus define charitable public recognition as occurring when the donor's donation decision will be made known to others. 
Based on this definition, an individual might perceive that they are donating because of their own choice to do so, but also because others are observing and evaluating the individual's behavior (Turner and Crisp 2007). ${ }^{1}$ Because of this, we propose that public recognition for charitable giving will lead to divergent reactions among those who have an independent (i.e., the self is viewed as separate and distinct from others) or interdependent (i.e., the self is viewed as connected with others) self-construal activated (Markus and Kitayama 1991; Singelis 1994). We predict that when an independent self-construal is activated people will donate less when the charitable act will be made public (vs. private). When an interdependent self-construal is activated this tendency will not be observed. Our framework proposes that these effects will emerge because those with an active independent self-construal wish to view their donation decision as driven by their own agentic choice rather than swayed by the influence of others, a motive that becomes particularly salient when the nature of the donation is public.

\section{PUBLIC RECOGNITION AND CHARITABLE GIVING}

Viewing charitable giving through an impression management lens, it may seem intuitive that making the donation public would increase donations. When a charitable donation is public, this increases awareness that the donor can be observed and evaluated by others (Turner and Crisp 2007). One consequence of this awareness may be an increase in the motive to impression manage or to present a positive image of the self to others (Froming et al. 1982; Kristofferson et al. 2014; Argo, White, and Dahl 2006). Indeed, past research suggests that public recognition can motivate charitable giving (Fisher and Ackerman 1998; Karlan and McConnell 2014; White and Peloza 2009) particularly because it acts as a symbolic reward, allowing the individual to present

\footnotetext{
${ }^{1}$ While charitable acts can also be privately recognized (e.g., with a private thank you note), we posit that it is the public element of public recognition that makes the focal effect emerge. We revisit this idea in study 3 .
} 
a positive view of the self to others (Grant and Mayer 2009; Lacetera and Macis 2010).

Kristofferson et al. (2014), for instance, demonstrate that an initial act of token support (i.e., signing a petition or joining a Facebook group) in public leads to less support on a subsequent task, which occurs because the initial act satisfies impression management motives when it is public versus private. Moreover, Winterich et al. (2013) demonstrate that among those who consider it highly important to convey their moral identity publicly, public recognition allows them to portray their prosocial nature to others, which increases donations. The implication is that a common driving mechanism of the positive effect of public recognition on donation amounts is the need to portray a positive self-image to others.

We propose that the straightforward desire to look good to others by appearing prosocial is not the only motive that can emerge under conditions that are highly public in nature. Instead, the public (vs. private) nature of the setting can also make it salient that one might be influenced by the expectations of others rather than by one's own agentic choice. As such, we draw on selfconstrual theory to suggest that charitable giving in response to public appeals will be moderated by the degree to which a more independent (vs. interdependent) self-construal is activated.

\section{The Role of Self-Construal}

Work on self-construal suggests that while the construct is often conceptualized at a cultural level, within cultures there are individual differences in terms of the extent to which people view the self as more independent or interdependent (Markus and Kitayama 1991). When considering the role of self-construal in predicting donation behavior, it is reasonable to assume that the socially connected nature of interdependents might lead them to generally be more inclined to make charitable donations. Indeed, some evidence of this pattern exists (Burton, Gore, and Sturgeon 2012; Moorman and Blakely 1995; Seo and Scammon 2014; Winterich and 
Barone 2011). There is also evidence, however, that US states that are more independent tend to give more (Kemmelmeier, Jambor, and Letner 2006; Conway et al. 2001), and that self-construal does not produce a main effect on giving (Duclos and Barasch 2014). Taken together, existing research has found mixed results regarding whether an interdependent or independent selfconstrual leads to more charitable behavior. This lends to consideration of potential moderators of the relationship between self-construal and donations. We suggest that the degree to which the donation context is more public or private is a relevant moderator of these effects.

When looking at the research regarding impression management and donations, one might expect that an independent self-construal increases donations in public settings (e.g., Ariely et al. 2009). Those who are independent are more likely to enhance the self (Markus and Kitayama 1991) and to engage in strategies that present the self in a positive light to others (Lalwani and Shavitt 2009; Wien and Olsen 2014). Thus, one possibility is that individuals who are under an independent self-construal will respond more positively to charitable requests that allow for some form of public recognition than to those that are relatively private in nature. However, we make a more counterintuitive prediction - that lower levels of charitable giving will be observed among those with an activated independent construal when the context involves public recognition compared to when the context is more private. This occurs because, among those with an activated independent self-construal, public recognition makes an agentic motive salient, leading them to want to freely make their own decisions based on their own motives and characteristics, while avoiding the influence of others. Given that the construct of self-construal is uniquely grounded in how the self is viewed with respect to others (Markus and Kitayama 1991; Singelis 1994) and relates to how people respond to social norms (Ji, Schwarz, and Nisbett 2000; White and Simpson 2013), we suggest this construct will moderate the impact of donation 
setting (i.e., public vs. private) on donations. While there are other cultural determinants of charitable behavior, such as power distance (Han, Lalwani and Duhachek 2017; Winterich and Zhang 2014), masculinity/femininity (Nelson et al. 2006), and uncertainty avoidance (Stojcic, Kewen, and Xiaopeng 2016), self-construal distinctively impacts how people perceive the influence of others. Because of this, we predict that those who have an independent selfconstrual activated will be most likely to assert their agency when the donation setting is public.

\section{Independent Self-Construal, Agency, and Public Charitable Giving}

Agency is the feeling that one can take motivated action towards desired outcomes in ways that allow for a sense of control and autonomy over one's own actions (Bandura 1989; Cutright and Samper 2014; Ryan et al. 1995; Ryan and Deci 2000). Research suggests that, for those with an independent self-construal, agency is an important value that reflects one's personal sense of ability and control, leading to choices that reflect internal needs and rights despite social pressure (Abele and Wojciszke 2007; Markus and Kitayama 1991). We propose that when an independent self-construal is active, people will want to a) be free to make their own choices without being influenced by others' opinions and expectations, and b) choose in a manner that is congruent with their own self-interest.

Notably, in a context where one's actions are publicly recognizable, individuals become aware of the pressure to conform to the expectations of others (Lerner and Tetlock 1999; Ratner and Kahn 2002; White and Peloza 2009). Because others will be aware that the potential donor knew the donation would be made public, donating may signal that the individual conformed to social pressure and expectations rather than relying on internal needs and goals (Miller 1999; Ratner and Miller 1998). As discussed earlier, being unduly influenced by external pressure is inconsistent with the desire for agency that characterizes an independent self-construal, a desire 
that should become especially salient when the donation is public. As a result of this inconsistency, we predict that an activated independent self-construal will lead people to donate less when their contribution will be publicly recognized as compared to when it is private.

Given this reasoning, factors that resolve the desire to assert agency should mitigate the observed effects. If the goal of making agentic decisions can be satiated in some way, then the tendency to decrease donations in public for those under an independent self-construal will be mitigated. For example, if the consumer learns that the donation act reflects an agentic goal, this should decrease the observed tendency for independents to donate less in public versus private. Furthermore, if a benefit to the individual self is made salient, then this tendency will be mitigated, and may even reverse as the donation would serve the donor's own self-interests. In contrast to an independent self-construal, an interdependent self-construal makes people feel more connected to others (Gardner et al. 1999). Given this, there are two potential ways a public context might impact charitable giving for those under an interdependent selfconstrual. First, the connected nature of an interdependent self-construal may lead people to want to donate for the "right" reasons. That is, they might donate less in public settings because they do not want to appear to donate merely to look good to others. Second, their connected nature may increase donations in public compared to private settings, as a public setting may make relationships with other people more salient. Given these two alternatives, it is difficult to make a specific prediction as their combination may lead to a null effect of the donation setting. Thus, we make the conservative prediction that under an interdependent self-construal, a public (vs. private) appeal to donate will not reduce donations. We examine this issue empirically in several studies, and additionally consider whether there is any general trend across all studies. 


\section{OVERVIEW OF THE CURRENT INVESTIGATION}

This research builds on the previous literature to further delineate the role public recognition can play in soliciting charitable donations (Fisher and Ackerman 1998; Karlan and McConnell 2014; White and Peloza 2009; Winterich et al. 2013). As such, it offers a number of important contributions. First, this research provides evidence of a moderator that may address some of the mixed findings about the relationship between self-construal and prosocial behavior. Second, this research contributes to work on the norm of self-interest (Holmes, Miller, and Lerner 2002; Miller 1999; Ratner and Miller 1998), which shows that individuals are often hesitant to engage in prosocial behaviors unless they can justify that such behaviors benefit the self. We demonstrate that the tendency to avoid the influence of others (and, instead, act in one's own interests) is high under an independent self-construal, especially when the actions are publicly conveyed to others. Third, previous research indicates that an independent self-construal can be associated with agentic motives, but has not demonstrated that this motive tends to become more activated in public settings. This is important as it qualifies the previously described association, showing when it is likely to be more salient and influence behavior.

Fourth, while this research does not purport to explain all previous findings, it may help answer the question of why previous research has often found a positive effect of public recognition on donations. In Karlan and McConnell (2014), the public aspect of the donation instructed participants that by donating they would "become a member of our Friend donor circle. Friends will be listed by name in the Dwight Hall Fall 2008 newsletter." In Lacetera and Macis (2010), people would "receive a reward for a donation in a public ceremony." One possibility, then, is that some public recognition manipulations may make people feel more connected with others, activating an interdependent self-construal. When greater donations have 
been observed in public versus private contexts, this might have been due in part to an activated interdependent self-construal interacting with the donation setting. We acknowledge that an interdependent self-construal could lead to increased donations in public, which is something that we will also test in our studies. Importantly, we orthogonally manipulate donation setting (public vs. private) and self-construal, which allows us to more clearly investigate how these factors interact to influence charitable donations.

Seven studies support our predictions through diverse operationalizations of our focal constructs. Studies 1 and 2 manipulate self-construal in field and lab experiments, respectively, to show that those with an activated independent self-construal are less likely to donate under conditions of public recognition (vs. private conditions). Study 3 measures self-construal and shows the mediating role of agentic motives, while accounting for the role of other cultural variables. Studies 4A and 4B both provide further evidence for the role of agentic motives by showing that the decrease in donations among independents in public is not observed if an agentic motive can be satiated. Study 5 shows that highlighting the benefits to the self, an additional element of how independents experience agency, also mitigates the observed effect. Finally, study 6 shows that the effect reverses when an impression management motive (i.e., to look prosocial to others) is made salient.

\section{STUDY 1}

In a field experiment, we manipulate self-construal via how marketing communications are presented, and manipulate public recognition by posting individuals' hand-written names in recognition of their support versus keeping their identity private. In addition, we track whether

people donate their own money to the cause and their actual donation amounts. We predict that 
when an independent self-construal is activated people will be less likely to donate when the donation will become public than when it will remain private. In contrast, this difference will be attenuated when an interdependent self-construal is activated.

\section{Method}

Participants and Design. One hundred and twenty participants took part in the study at the University of British Columbia campus. Participants were 55\% male, with a mean estimated age of 24 ( $\mathrm{SD}=8.54$, range 18-60). The design was a 2 (self-construal: independent vs. interdependent) x 2 (setting: public vs. private) between-subjects design.

Procedure. The study was conducted prior to Remembrance Day in Canada in conjunction with the Poppy campaign that honors Canada's veterans. The study took place outside a coffee shop on campus. Previous research demonstrates that self-construal can be primed via wording in a story presented before the focal task (Brewer and Gardiner 1996; White and Argo 2011) and via marketing communications (White and Simpson 2013). Thus, selfconstrual was manipulated by both the signage and the words spoken by the research assistant to solicit donations from passersby. The assistant stopped passersby and motioned to a poster that communicated via singular pronouns in the independent condition ("I remember...") or plural pronouns in the interdependent condition ("We remember..."; see app. A). In addition, the assistant appealed for a donation using independent (or interdependent) wording: "Please donate to support your (our) veterans. You (We) can honor those who have given so much, and help by donating. Show that you (we) remember."

In the private setting condition, participants were told "if you decide to make a donation, you can place it in the envelope to make sure it is private," and were given an envelope to place the donation inside. In the public condition, a large flip chart was displayed on a stand, and 
participants were told that if they "decided to make a donation, you can write down your name on the sheet and we will display all of our donors on the wall." To make the conditions comparable, public condition participants also received an envelope to place their donation in. Each of the four conditions was run in one-hour time blocks (and each was run for a total of 2 hours). The dependent variables were recorded by the research assistants as 1) willingness to donate (whether participants donated: yes or no) and 2) the amount participants donated (in dollars and cents). All funds were donated to the Canadian Poppy Fund.

\section{Results and Discussion}

Willingness to Donate. We removed one participant from the dataset who donated $\$ 20$ on behalf of six people. Thus, the final analyses were conducted on 119 individuals. In a logistic regression, willingness to donate was regressed on self-construal, setting, and their interaction $(\mathrm{Wald}=4.55 ; \beta=-1.66, p=.03$; see fig. $1 \mathrm{~A})$. As anticipated, in the independent condition, people were marginally less likely to donate when the setting was public (27.3\%) than private $(48.5 \% ; p=.06)$. In the interdependent condition, willingness to donate did not differ significantly based on whether the setting was public $(61.9 \%)$ or private $(43.8 \% ; p=.16)$.

Donation Amount. Following previous research in the donation domain, the data were log transformed because they were positively skewed and contained a number of zeros, although raw data means are presented to ease interpretation (see MacDonnell and White 2015). There was a marginally significant interaction between self-construal and setting on donation amount $(F(115)$ $=3.39, p=.07$; fig. 1B). In the independent condition, people donated directionally less when the setting was public $(M=.75, \mathrm{SD}=1.53)$ than when it was private $(M=1.32, \mathrm{SD}=1.74$; $F(115)=2.64, p=.10)$. In the interdependent condition, people donated directionally, but not 
significantly, more when the setting was public $(M=1.50, \mathrm{SD}=1.91)$ than when it was private $(M=1.18, \mathrm{SD}=1.91 ; F(115)=1.05, p=.31)$.

Using a naturalistic field experiment, study 1 demonstrates that activating an independent self-construal may decrease people's monetary donations when these contributions will be publicly recognized. Admittedly, some results were only marginally significant, possibly because of noise resulting from running a study in the field. For example, our research assistants reported that some participants were in a rush and some had already donated directly to the cause. To address these issues, in the following studies we test our predictions in more controlled settings.

\section{STUDY 2}

Study 2 takes place in a laboratory setting, manipulating self-construal and again appealing to participants for a charitable donation by highlighting that the donation will be kept private or made public. We predict that when an independent self-construal is activated people will donate less when the donation is public than when it is private. This difference should be attenuated when an interdependent self-construal is activated.

\section{Method}

Participants and Design. Participants were 242 undergraduate students attending the University of Delaware who participated in exchange for partial course credit. Participants were $45 \%$ male, with a mean age of $19.78(\mathrm{SD}=1.98$, range $18-24)$. The study was a 2 (self-construal: independent vs. interdependent) x 2 (setting: public vs. private) between-subjects design.

Procedure. Participants were first asked to complete a task that ostensibly measured verbal processing. They were asked to read a short story and instructed to click on pronouns appearing in the story text. The text in the independent condition contained only the "I," "me," 
and "my," pronouns, whereas the text in the interdependent condition contained only "we," "us," and "our." While this self-construal manipulation has been used successfully in previous research (e.g., Gardner, Gabriel, and Lee 1999; White and Argo 2011; White, Argo, and Sengupta 2012), we confirmed its efficacy with a pretest (see web appendix).

All participants were then exposed to a fictional appeal from the United Way of America (app. B). Setting was manipulated with statements viewed prior to the donation request: those in the public condition were told "Please note, your contribution will be public and your name will be listed as a donor on the fundraising campaign of your regional United Way website." Those in the private condition were told "Please note, your contribution is completely anonymous and confidential." Again, a pretest was conducted to confirm the efficacy of the setting manipulation and that our manipulation of setting did not impact self-construal (web appendix).

Finally, in the main study, participants indicated the amount that they would be willing to donate to the United Way (percentage of a $\$ 2$ bonus payment) as the dependent variable. Actual donations were made to the United Way of America on behalf of participants.

\section{Results and Discussion}

The data were skewed and were therefore log transformed for analysis. There was only a significant interaction between self-construal and setting on donations $(F(1,238)=5.99, p=.02$; see fig. 2). In the independent condition, people donated less when the setting was public $(M=$ $68.51, \mathrm{SD}=43.75)$ than when it was private $(M=83.35, \mathrm{SD}=35.78 ; F(1,238)=4.70, p=.03)$. In the interdependent condition, people donated similar amounts when the setting was public $(M$ $=86.15, \mathrm{SD}=31.78)$ and private $(M=77.45, \mathrm{SD}=39.13 ; F(1,238)=1.69, p=.20)$.

Study 2 provides further support for our predictions by demonstrating that an independent self-construal can be activated via an experimental manipulation and influence donations. This 
occurs in a way that people donate less when an independent self-construal is activated and the donation will be made public. In study 3 we sought to provide evidence for this effect when selfconstrual is measured, along with evidence that an agentic motive drives the effect.

\section{STUDY 3}

Study 3 tests our conceptualization in a different way by measuring self-construal instead of manipulating it. We predict that participants who are relatively more independent will donate less when the donation is public than when it will remain private while this difference should be attenuated for those with a more interdependent self-construal. We also sought to demonstrate the mediating role of agentic motives. To do so, we drew from psychological literature on autonomy and agency (e.g., Deci and Ryan 1985; Ryan and Deci 2000). According to Deci and Ryan, to assert agency means having a sense of free will to make one's own decisions and acting out of one's own interests and values (see also Chen et al. 2015). We use this conceptualization to test for the mediating role of agentic motives in this study. In addition, we generalize the effect to a new organizational and charitable context, using a less socially-oriented charity.

Moreover, we measured additional cultural dimensions (power distance, uncertainty avoidance, masculinity-femininity, long-term orientation) to examine their possible influence on the results. Finally, we wished to confirm that it is the public nature of the recognition, rather than any form of recognition, that is driving the observed effects. We did so by offering all participants a form of private recognition - a personal thank you. We reasoned that if the effect failed to replicate for those with an activated independent self-construal under conditions of private recognition, this would imply that the effect is driven by recognition and not by the 
public nature of the setting. However, if it is the public nature of the recognition that is driving the effects, we should observe our focal effect in the public (but not private) condition.

\section{Method}

Participants and Design. Participants were 163 MTurk workers who received payment to participate in the study. Participants were $56 \%$ male, with a mean age of 33.88 (SD $=9.89$, range 19-69). We manipulated donation setting at two between-subject levels (public vs. private) and measured self-construal as an individual variable.

Procedure. Participants viewed a fictional appeal from the American Society for the Prevention of Cruelty to Animals (ASPCA; see app. C). They next read that there would be five bonus draws for $\$ 20$, and were told that the researchers would like to give those who win the draws the opportunity to support the ASPCA. Prior to being asked to donate, participants viewed the same manipulation of setting used in study 2 , and then completed manipulation checks to be sure they understood the nature of the setting: "Did you learn that your donation decision would be shared with others?," "To what degree do you think others think your decision to donate or not would be made public?," and "To what degree does the donation situation today create pressure from others to donate?," (all 1-7, not at all - very much so). All participants were then asked how much of this bonus draw prize they would donate, first viewing that "If you do choose to donate, you will see a letter of thanks from ASPCA at the end of the survey." Thus, there would be recognition for donating in both the private and public conditions. They then used a slider (0-100\%) to specify their donation. This percentage was our dependent variable.

Participants then completed a 24-item self-construal measure (Singelis 1994; strongly disagree - strongly agree on seven-point scales) and four items measuring agentic motives (Chen et al. 2015; Ryan and Deci 2000) adapted to the donation context: "The donation request made 
me feel a sense of choice and freedom with regards to the donation decision," "The donation request made me feel that my donation should reflect what I really want to do," "The donation situation made me feel that my donation choice should express who I really am," and, "In considering the donation request, I felt like my freedom to choose what to donate was restricted" (all 1-6, not at all - very much so). The first three items were negatively correlated to the fourth and thus reversed, and then all four were averaged to form an index of agentic motives $(\alpha=.80)$. Participants also completed measures of Hofstede's other dimensions of cultural values (Hofstede 2001; Yoo, Donthus, and Lenartowicz 2011), including five items measuring power distance $(\alpha=.88)$, three items measuring uncertainty avoidance $(\alpha=.67)$, two items measuring masculinity-femininity ( $\alpha=.80$ ), and four items measuring long-term orientation $(\alpha=.66)$.

\section{Results and Discussion}

Manipulation Checks. Participants in the public condition had higher responses than those in the private condition to each setting manipulation check question: 'Did you learn that your donation decision would be shared with others?' $\left(M_{\text {public }}=6.30, \mathrm{SD}=1.84 ; M_{\text {private }}=1.85\right.$, $\mathrm{SD}=1.67 ; t(161)=15.81, p<.001)$; 'To what degree do you think others think your decision to donate or not would be made public?' $\left(M_{\text {public }}=5.62, \mathrm{SD}=1.91 ; M_{\text {private }}=1.90, \mathrm{SD}=1.70\right.$; $t(161)=13.14, p<.001)$; and, 'To what degree does the donation situation today create pressure from others to donate?' $\left(M_{\text {public }}=4.41, \mathrm{SD}=2.06 ; M_{\text {private }}=2.12, \mathrm{SD}=1.65 ; t(161)=7.84, p<\right.$ $.001)$. The manipulation of setting did not influence self-construal $(p=.25)$.

Donations. The self-construal measure was calculated such that higher scores reflect higher levels of independence. As donations and the mediator were positively skewed, both were $\log$ transformed. Donation was regressed on self-construal, setting, and their interaction, and showed a marginal interaction between self-construal and setting $(\beta=-.01, p=.075)$. Among 
independent participants (i.e., $+1 \mathrm{SD}$ above the self-construal mean) a public (vs. private) setting of the charitable behavior led to lower donation amounts $\left(\mathrm{B}_{\mathrm{JN}}=-.38, S E=.16, p=.02\right.$; see fig. 3). Among interdependent participants there was no significant effect of setting (at 1 SD below the mean $\left.\mathrm{B}_{\mathrm{JN}}=.04, S E=.16, p=.81\right)$.

Mediation by Agency. Our framework proposes that for independents agentic motives become more salient when the donation setting is public, which will mediate the effect of the interaction on donations. Overall the model predicted agentic motives $(F(1,159)=5.94, p<$ $.001)$, and a main effect of setting $(t=2.25, p=.03)$ was qualified by a significant interaction between self-construal and setting on agentic motives $(t=2.07, p=.04)$. For independents ( $1 \mathrm{SD}$ above the self-construal mean), a public setting made agentic motives more salient $\left(\mathrm{B}_{\mathrm{JN}}=.18, S E\right.$ $=.05, p<.001)$ whereas for interdependents (1SD below the self-construal mean) setting did not impact agentic motives $\left(\mathrm{B}_{\mathrm{JN}}=.05, S E=.05, p=.33\right)$. To test for mediation, we used PROCESS model 7 (5000 bootstrap samples; Hayes 2013). The interaction of self-construal and setting predicted agency $(t(159)=2.07, p=.04)$ and agency predicted donations $(t(159)=-3.15, p<$ .01). When agentic motives was added to the model examining the effect of the interaction on donations, the effect of the interaction became nonsignificant $(p=.18)$. Supporting our predictions, the pathway from setting to donations through agentic motives was significant and did not include zero for independents (CI: -.32 to -0.05), supporting mediation. The pathway was not significant for interdependents (CI: -0.14 to 0.03 ).

Additional Measures. To understand the influence of additional cultural constructs derived from the charitable giving literature, we conducted a regression model with selfconstrual, setting, their interaction, as well as each of the constructs listed above (scaled items averaged) and their interactions with setting. The interaction of self-construal and setting 
predicting donations remained significant $(t(151)=-2.48, p=.01)$ while all other constructs and their interactions with setting were nonsignificant (all $p$ 's $>.12$ ).

Study 3 supports our predictions by demonstrating that independents are less likely to donate in public than in private, and that this effect is mediated by agentic motives. Finally, the results demonstrate that the effects are due to the public nature of the recognition, and not the recognition itself, given that offering private recognition did not mitigate the effect. Note that a replication of the basic effect demonstrated here can be found in the web appendix.

\section{STUDY 4A}

While the previous study demonstrated mediational evidence of our proposed mechanism, we also want to provide process evidence using a moderation-of-process approach (Bullock, Green, and Ha 2010; Spencer, Zanna, and Fong 2005). Recall that our conceptualization predicts that when an independent self-construal is active, people are driven by an agentic motive to a) be free to make their own choices without being influenced by others' opinions and expectations, and b) choose in a manner that is congruent with their self-interest. In studies $4 \mathrm{~A}$ and $4 \mathrm{~B}$, we focus on the former, and tell half of participants that their donation decision is their own agentic choice. We predict when independents are given a public charitable request, resolving an agentic goal will increase donations as compared to a control condition. When independents are given a charitable request in private, agency will not be threatened in any way and an agentic goal should not impact donations. We also employ a new manipulation of public-private setting to provide further evidence for the generalizability of our effect.

\section{Method}


Participants and Design. Participants were 243 undergraduate students from University of Miami who participated in exchange for partial course credit. Participants were $51 \%$ male, with a mean age of 20.07 ( $\mathrm{SD}=2.13$, range $18-37)$. This was a 2 (agentic motive: control vs. satiation) x 2 (setting: public vs. private) between-subjects design. As our focus was on the role of agentic motives, we activated an independent self-construal for all participants.

Procedure. We used the same task from study 2 to activate an independent self-construal for all participants. To manipulate setting, participants in the private setting condition were told "Please remember that all of your responses will be completely anonymous and confidential," while participants in the public setting condition were told "We are particularly interested in hearing about your intentions and evaluations regarding behaviors. To this end, after you have reported your attitudes and evaluations you will be asked to discuss your responses with other participants in the room today." While this manipulation has been validated in previous research (White and Peloza 2009; White, Simpson, and Argo 2014), we again confirmed its efficacy in a pretest (see web appendix).

Participants were then exposed to the same appeal used in study 2 , followed by a request to donate. Prior to indicating how much they would donate, on the same page as the donation request, participants viewed the agentic motive manipulation. The control condition included no additional information, while the agentic motive satiation condition stated that "We understand that you are making your own decision, and that this decision represents your own goals." Finally, they read that there would be five $\$ 20$ dollar draw prizes that they could win for participating, and asked "If you won one of these $\$ 20$ prizes, how much of the prize money would you like to donate to the United Way?," using a slider (\$0-20) to specify their donation.

\section{Results}


There was only an interaction between agentic motive and setting on donations $(F(1,239)$ $=5.52, p=.02$; see fig. $4 \mathrm{~A}$ ). In the control condition, people donated less when the setting was public $(M=\$ 9.55, \mathrm{SD}=6.94)$ than when it was private $(M=\$ 12.54, \mathrm{SD}=6.57 ; F(1,239)=$ $5.43, p=.02)$. In the agentic motive satiation condition, people donated similar amounts when the setting was public $(M=\$ 12.09, \mathrm{SD}=6.89)$ and private $(M=\$ 10.83, \mathrm{SD}=7.79 ; p=.32)$. As predicted, when the donation was public, people donated more in the agentic motive satiation condition than in the control condition $(F(1,239)=3.97, p=.05)$. There was no effect of agency when the donation was private $(p=.19)$. We further discuss the results below with study $4 \mathrm{~B}$.

\section{STUDY 4B}

The goal of study 4B is to provide additional evidence for the role of an agentic motive. We achieve this goal by using a new manipulation of agentic motive satiation that reflects having made one's own choice, independent of the influence of others. In addition, we provide further

evidence for the mediating role of one's perception of agency in the donation situation. While in study 3 we measured the extent to which a given setting made an agentic motive salient (i.e., a public setting makes the motive more salient), here we measured the extent to which a given setting satisfied a salient agentic motive (i.e., a public setting should satisfy it less). Mediation would indicate that donations decrease in a public setting because the request makes people feel like they are being told to do what others want (i.e., it does not satisfy an agentic motive). Finally, as one possibility is that the causes we have used in some of the previous studies may have been congruent with interdependent values, in this study we demonstrate our effect with an alternative, educational cause (Kemmelmeier et al. 2006; Nelson et al. 2006).

\section{Method}


Participants and Design. Participants were 391 undergraduate students attending University of Miami who participated in exchange for partial course credit. Participants were $56 \%$ male, with a mean age of $19.71(\mathrm{SD}=1.88$, range $18-42)$. All participants were primed with an independent self-construal, and the design was a 2 (setting: public vs. private) x 2 (agentic motive: control vs. satiation) between-subjects design.

Procedure. Self-construal and setting were manipulated as in study 4A. All participants were then exposed to a fictional charitable appeal (Pathways to Education; see app. D). Prior to indicating how much they would donate, participants viewed the agentic motive satiation manipulation. The control condition included no additional information, while the agentic motive satiation condition stated that "We know this decision will not represent the influence of others, you are doing what you choose to do." Participants then read "If you won one of these $\$ 20$ prizes, how much would you donate to Pathways to Education?" and indicated their donation amount (\$0-20). Following the donation request, we measured perceived agency as a mediator with the following question: "The donation situation made me feel like I was being told to do what I wanted, not what others wanted (1-6, Do what others wanted - Do what I wanted).

\section{Results and Discussion}

Donations. There was a main effect of setting $(F(1,387)=6.89, p=.01)$, qualified by an interaction between agentic motive and setting on donations $(F(1,387)=5.63, p=.02$; see fig. 4B). In the control condition, people donated less when the setting was public $(M=\$ 8.22, \mathrm{SD}=$ 6.84) than when it was private $(M=\$ 11.79, \mathrm{SD}=7.30 ; F(1,387)=12.71, p<.001)$. In the agentic motive satiation condition, people donated similar amounts when the setting was public $(M=\$ 10.61, \mathrm{SD}=6.72)$ and private $(M=\$ 10.79, \mathrm{SD}=7.31 ; F(1,387)=.03, p=.86)$. As predicted, when the donation was public, people donated more in the agentic motive satiation 
condition than in the control condition $(F(1,387)=5.38, p=.02)$. There was not an effect when the donation was private $(p=.31)$.

Mediation by Agency. There was a main effect of agentic motive $(F(1,387)=4.95, p=$ $.03)$, a main effect of setting $(F(1,387)=5.40, p=.02)$, and a marginally significant interaction between agentic motive and setting on the mediator $(F(1,387)=3.34, p=.068)$. In the control condition, people reported lower perceived agency when the setting was public $(M=3.22, \mathrm{SD}=$ 1.74) than when it was private $(M=3.95, \mathrm{SD}=1.68 ; F(1,387)=8.81, p<.01)$. In the agentic motive satiation condition, people perceived similar levels of agency when the setting was public $(M=3.93, \mathrm{SD}=1.67)$ and private $(M=4.02, \mathrm{SD}=1.83 ; F(1,387)=.12, p=.73)$. As predicted, when the donation was public, those in the agentic motive satiation condition reported greater perceived agency than those in the control condition $(F(1,387)=7.89, p<.01)$.

To test for mediation, we used PROCESS model 7 (5000 bootstrap samples; Hayes 2013). The interaction of agentic motive and setting predicted perception of agency $(p<.01)$ and perception of agency predicted donations $(p<.001)$. When perception of agency was added to the model examining the effect of the interaction on donations, the effect of the interaction became nonsignificant $(p>.05)$. Supporting our predictions, the pathway from setting to donations through the agency mediator was significant and did not include zero in the control agentic motive condition (CI: -2.12 to -0.46 ), supporting mediation. The pathway was not significant and included zero in the agentic motive satiation condition (CI: -0.95 to 0.70$)$, not supporting mediation.

Taken together, studies 4A and 4B provide further evidence for the role of agentic motives in determining the previously observed effects. Under an independent self-construal, when it was highlighted that people were acting in line with their agentic motives the difference 
in donations between the public and private conditions was mitigated. Moreover, study 4B demonstrated that when an independent self-construal is activated and the donation will be public, people feel as if the donation context does not satisfy their agentic motive (i.e., it involves doing what others want), which decreases donations. Across both studies 4A and 4B, there was no effect of the agentic motive satiation manipulation when the donation was private. This is consistent with our theorizing as private contexts likely do not make consumers who are under an independent self-construal feel that their behaviors are being influenced by others.

\section{STUDY 5}

We propose that agency can be asserted by both a) freely making one's own choices and b) choosing in one's self-interest. While studies 4A and 4B addressed "a," study 5 addresses the role of self-interest. To achieve this goal, half of the participants were informed that making a public donation brings benefits for the self. If an independent self-construal leads people to donate less in public because of their agentic motives, highlighting benefits for the self should make donating in public more appealing to these individuals. Thus, in this condition, we expected independent participants to donate more in public than in private.

\section{Method}

Participants and Design. Participants were 342 undergraduate students at University of Miami who participated in exchange for partial course credit. Participants were $45 \%$ male, with a mean age of $19.88(\mathrm{SD}=1.78$, range $17-26)$. The design was a 2 (benefit: control vs. self) x 2 (self-construal: independent vs. interdependent) x 2 (setting: public vs. private) between-subjects design. 
Procedure. Self-construal and setting were manipulated as in studies 4A and 4B. All participants were then exposed to the same appeal from the United Way of America used in studies 2 and 4A. Prior to indicating how much they would donate, participants viewed the benefit manipulation. The control condition included no additional information, while the selfbenefit condition stated that "Recent analysis of donation data and people's perceptions indicates that donations that are made public (others end up knowing about it) are the ones that benefit the donor the most in terms of what they end up getting back for it." Finally, participants saw "If you won one of these $\$ 20$ prizes, how much would you donate to the United Way?” and indicated a dollar amount (0-20).

\section{Results and Discussion}

Again, the donation variable data were skewed and we therefore used a log transformation in our statistical analyses. There was a 3-way interaction of the benefit, selfconstrual, and setting factors on donations $(F(1,334)=4.49, p=.03$; see fig. 5$)$. In the control benefit condition, there was an interaction between self-construal and setting $(F(1,175)=4.97, p$ $=.03)$. In the independent condition, people donated less when the donation was public $(M=$ $\$ 7.02, \mathrm{SD}=6.64)$ than when it was private $(M=\$ 9.44, \mathrm{SD}=6.76 ; F(1,334)=5.51, p=.02)$. In the interdependent condition, people donated similar amounts when the donation was public $(M$ $=\$ 8.28, \mathrm{SD}=7.73)$ and private $(M=\$ 6.46, \mathrm{SD}=6.23 ; F(1,334)=1.32, p=.25)$.

In the self-benefit condition, however, there was not an interaction $(F(1,159)=.53, p=$ $.47)$, but only a main effect of setting $(F(1,159)=17.28, p<.01)$. Overall participants donated more when the donation was public $(M=\$ 11.07, \mathrm{SD}=6.82)$ than when it was private $(M=$ $\$ 7.04, \mathrm{SD}=6.34 ; F(1,334)=15.46, p<.001)$. 
Study 5 demonstrates that highlighting that a public donation is in line with an agentic goal reverses the results from the previous studies for an independent self-construal. When a self-benefit is salient the effect of setting is no longer moderated by self-construal, and those with an activated independent self-construal are more likely to donate in public versus private. While these findings contribute to our understanding of how an independent self-construal leads people to act per their agentic motives, one potential criticism of this study is that the self-benefit manipulation may have been heavy-handed, leading to a demand effect. However, consistent with our conceptualization, we do not observe participants uniformly responding more positively to self-benefits across all conditions. Importantly, the effect of the self-benefit manipulation varied across public $(t(173)=-3.33, p<.001)$ and private conditions $(t(178)=1.16, p=.25)$.

Also note that, in the control condition, donations in private were higher among those in the independent compared to the interdependent condition. When considering this in light of the other studies that allow for this comparison (studies 1 and 2), this is the only study in which this difference was significant. One possible reason for this result is that the manipulation in the current study might have made the private setting particularly salient. Given that previous research using this same manipulation shows that private settings make self-benefits for donating salient (White and Peloza 2009), this may have increased donations in the independent (vs. interdependent) self-construal, private setting condition.

\section{STUDY 6}

Study 6 explores another condition under which people who are under an independent self-construal will be more inclined to donate in public. Given that previous research (Ariely et al. 2009; Karlan and McConnell 2014) has demonstrated that an impression management motive 
may increase donations, in study 6 we examine the moderating role of an impression management goal to look like a prosocial and helpful person. We predict that activating this impression management motive will only have an influence on donations in a public setting, making those with an active independent self-construal donate more in public than in private.

\section{Method}

Participants and Design. Participants were 120 undergraduate students from University of Miami who participated in exchange for partial course credit. Participants were $65 \%$ male, with a mean age of $19.34(\mathrm{SD}=1.63$, range $18-29)$. All participants were primed with an independent self-construal, and the design was a 2 (impression management motive: control vs. salient) x 2 (setting: public vs. private) between-subjects design.

Procedure. Self-construal and setting were manipulated as in study 5. All participants were then exposed to the fictional charitable appeal from "Pathways to Education" used in study 4B. Prior to indicating donation intentions, participants received the impression management manipulation: "We are interested in how people make donation decisions with a certain goal in mind. Please imagine that you have the goal to demonstrate to others that you are helpful and prosocial. Please keep this goal in mind as you make your decision about the donation." Those in the control condition received no additional information. Finally, participants responded to "How likely would you be to donate to Pathways to Education?," "How willing would you be to donate to Pathways to Education?," and "How inclined would you be to donate to Pathways to Education?" (all 1 - not at all to 7 - very much so). The items were averaged to form the dependent variable $(\alpha=.95)$ of donation intentions.

\section{Results and Discussion}


There was a main effect of impression management on donation intentions $(F(1,116)=$ $4.10, p=.05)$, qualified by an interaction between impression management and setting $(F(1,116)$ $=9.09, p<.01$; see fig. 6 ). In the control condition, participants reported lower intentions to donate when the setting was public $(M=3.03, \mathrm{SD}=1.66)$ than when it was private $(M=3.89$, $\mathrm{SD}=1.80 ; F(1,116)=3.79, p=.05)$. In the impression management condition, however, participants reported higher donation intentions in public $(M=4.60, \mathrm{SD}=1.74)$ than in private $(M=3.58, \mathrm{SD}=1.63 ; F(1,116)=5.38, p=.02)$.

Study 6 demonstrates that when the donation appeal highlights an impression management motive to appear prosocial and helpful to others, those with an independent selfconstrual activated donate more in public vs. private. These results are consistent with previous research on the relationship between impression management and donations, contributing to our understanding of when individuals might be more or less likely to impression manage (Ariely et al. 2009; Winterich et al. 2013). We discuss the implications of our findings next.

\section{GENERAL DISCUSSION}

Public recognition of charitable contributions is widely used by charities to encourage donations. The current research provides converging evidence that public recognition is not a universally successful strategy for engaging individual donors, and that it can backfire when an independent self-construal is activated. While several previous findings have garnered insights into when public recognition might be more or less effective (Fisher and Ackerman 1998; Karlan and McConnell 2014; Kristofferson et al. 2014; White and Peloza 2009; Winterich et al. 2013), and that people do not always donate for altruistic reasons (Bendapudi et al. 1996; Holmes et al. 2002), our research adds a nuanced perspective. We demonstrate that an independent self- 
construal, associated with agentic motives, leads consumers to want to make their own choices and avoid being unduly influenced by the opinions and expectations of others. The result is a decreased willingness to donate among those under an independent self-construal when the donation will be publically recognized versus kept private. Notably, the effect emerges under conditions where the donation behavior is made public because these are the very conditions under which people might feel their agency is compromised. We show evidence for this effect using consequential consumer donation behaviors and different ways of operationalizing both self-construal and donation setting.

We provided support for this conceptualization in the field (study 1) and in the lab (study 2), showing that those with an independent self-construal are less likely to donate when they will be publically recognized for donating. This effect occurs among those who have been primed with an independent self-construal (studies 1 and 2) and naturally with individuals who have a more independent self-construal (study 3). We provide evidence that the effect is driven by people who are under an independent self-construal wanting to maintain their agency. In particular, the effect is mediated by the salience of agency motives (studies 3 and 4B), and moderated by the opportunity to satisfy agency motives (studies 4A and 4B) and act in one's self-interest (study 5). Finally, examining only the effect on an independent self-construal, making an impression management motive salient reverses the effect, leading to more donations in public than in private (study 6).

\section{Theoretical Contribution}

The charitable giving literature finds that individuals are generally inclined to donate more in public (vs. private) contexts and suggests that this is often driven by the desire to present a positive self-image to others (Ariely et al. 2009; White and Peloza 2009; Winterich et al. 
2013). In contrast to this past work, the current research demonstrates that individuals with an active independent self-construal may not always be motivated to present a prosocial image of the self to others and may instead seek to satisfy agentic goals in public settings. This is a very intriguing result given that research suggests that independent selves are primarily motivated to enhance the self (Markus and Kitayama 1991) and engage in strategies that allow for positive impression management (Wien and Olsen 2014). Our results suggest that under public conditions where both impression management concerns (i.e., to appear prosocial to others) and agentic concerns (i.e., to freely make one's own decisions) have the potential of being activated, independent consumers are in fact more driven by the desire for agency. This is consistent with findings that self-construal predicts self-presentation goals, and specifically that independents tend to value presenting agentic qualities (e.g., competent, self-reliant, capable) more so than presenting themselves as behaving in a socially appropriate manner (Lalwani and Shavitt 2009). It appears, then, that agency is primary for independent consumers and it is only when a very strong impression management goal is salient that the desire to present a positive, prosocial self will override the desire for agency (as we saw in study 6).

Our research also provides insight into the role of self-construal in charitable giving. We find support for the notion that public recognition can indeed be an effective strategy in garnering donations, but we also highlight that this occurs mostly when an interdependent selfconstrual is activated. While we discussed in the introduction that two countervailing motives (presenting as altruistic and connectedness) may have made any effects of public setting for interdependents difficult to detect, our studies found a consistent pattern of higher donation amounts for interdependents in public versus private. We thus pooled t-values (Winer 1971; see also White et al. 2014) to further understand this aspect of the findings. The result is a meta- 
analysis of the effect across studies where interdependent construal was included (study 3 and control conditions of studies 1,2, and 5). Considering the data collectively, the meta-analysis revealed that the interdependent effect was significant $(\mathrm{z}=1.60 ; p=.05)$. Thus, our data support the existing notion in the public recognition literature that the strategy can be effective, but also provides new insight into when it may not be effective. As predicted, a meta-analysis across our independent self-construal data (studies 1, 2, 3, 4A/B, 5, and 6 control) also shows that an active independent self-construal leads to less donations in public than in private $(\mathrm{z}=5.41 ; p<.001)$. These findings also add to the discussion of the nature of the relationship between self-construal and donation behavior (Duclos and Barasch 2014; Winterich and Barone 2011), as our data indicate that this relationship can be moderated by the nature of the setting.

The current research also deepens the broader understanding of the relationship between agentic motives and situational demands, demonstrating that, among interdependents, agency is more likely to be salient in public as opposed to private settings. To our knowledge, this is the first work to show that agency concerns can become paramount under public (vs. private) conditions. Finally, our work builds on the literature on the norm of self-interest (Miller 1999). Our findings are consistent with the theorizing of the norm of self-interest, and suggest that this norm is particularly relevant when an independent self-construal is activated. Importantly, our findings suggest that a public setting is central in activating the inclination assert agency and to avoid being influenced by others. This implies that adhering to the norm of self-interest is not straightforward and universal, as setting and self-construal interact to determine the degree to which self-interested behaviors are observed.

\section{Practical Implications and Directions for Future Research}


The current research suggests practical implications for charitable organizations when soliciting donations. One possibility would be to activate an interdependent self-construal when appealing to donors using public recognition. For example, campaigns often highlight how "we" can make a difference. Our study 1 suggests that such wording within charitable appeals might be used to activate an interdependent self-construal, making people more likely to donate and to contribute more in response to public requests for donor support. Activating an independent selfconstrual, in order to profit when donations are private, could also be done in the wording of the campaign in ways that highlight personal pronouns (i.e., "I" and "my") as our work suggests that appeals themselves can be used to manipulate self-construal (study 1). Charitable marketers should also be cognizant of how the communications and contexts they use can alter donors' perceptions of how public or private a donation is. Subtle differences in the donation setting or the type of appeal made can impact how publicly recognizable donations are, which may impact consumer charitable giving.

To avoid negative outcomes when using public recognition as a tactic, charitable organizations could also consider reminding independent consumers that the charitable donation is their own agentic, free choice. This might be done through statements, such as we have demonstrated, or through empowering consumers with a choice regarding whether their donation becomes public, although more research would be needed to fully understand the implications of doing so (e.g., Wang and Tong 2015). Alternatively, organizations might want to consider making an impression management motive salient, as our work shows preliminary evidence that activating a specific prosocial goal can counter an agentic motive.

One other direction for future research might be to examine the impact of donation appeals that strongly highlight the benefit to others (i.e., White and Peloza 2009). Our results 
suggest that an interdependent (vs. independent) self-construal may be more congruent with an other-benefit appeal when the setting is public. Whether an appeal promotes benefits to others is an interesting avenue to explore, as it is possible that the type of appeal (Han et al. 2017; Park and Lee 2015; Scholsser and Levy 2016; Kulow and Kramer 2016) that is made salient could moderate our results. For instance, under public conditions, it may be that making salient that the donation would benefit others intensifies negative reactions of those under an independent selfconstrual and enhances the positive reactions of those under an interdependent self-construal. Future research could explicitly vary self-benefits, other-benefits, or no benefits and look at how this impacts charitable giving when an independent or interdependent self-construal is activated.

One limitation of the present research is that we only explore monetary charitable giving intentions and behaviors. Given that organizations also use public recognition to encourage donations of time (i.e., volunteerism) and research shows that giving money and time have distinct psychological consequences (MacDonnell and White 2015), future research could examine whether our effect holds in the context of public recognition of alternative forms of charitable contributions. For instance, giving time (vs. money) perhaps reflects a greater commitment to a given cause, which might lead to a decreased desire for agency. Moreover, because giving time may garner more benefits to the self (e.g., experience, skills, networking connections, etc.), the observed effect might be mitigated when giving time (vs. money).

In conclusion, we have taken an additional step toward demonstrating that public recognition can potentially lead to undesirable donation consequences. With this demonstration comes the possibility of further understanding these effects in order to mitigate them, increasing donation behavior in ways not yet explored. While we have shown some of the ways in which this can be accomplished, we hope these findings will stimulate further research in this area, 
exploring the theoretical and practical consequences of self-construal and public recognition on donation behavior. 
Data collection summary: The second author supervised collection of data for the first study by research assistants at the University of British Columbia in the fall of 2015. The first and second authors jointly analyzed these data. The data collection for study 2 was conducted at University of Delaware and managed jointly by the first and third authors, with analyses completed jointly by the first and third authors. Study 3 (and replication, see web appendix) were conducted on MTurk between spring 2015 and spring 2017 and managed jointly by the first and third authors, with analyses completed jointly by the first and third authors. Studies 4A-6 were conducted by a lab assistant at the University of Miami, supervised by the first and third authors, between spring 2015 and spring 2017. Analyses of the data were conducted jointly by all three authors. 


\section{APPENDIX A - STIMULI USED IN STUDY 1}

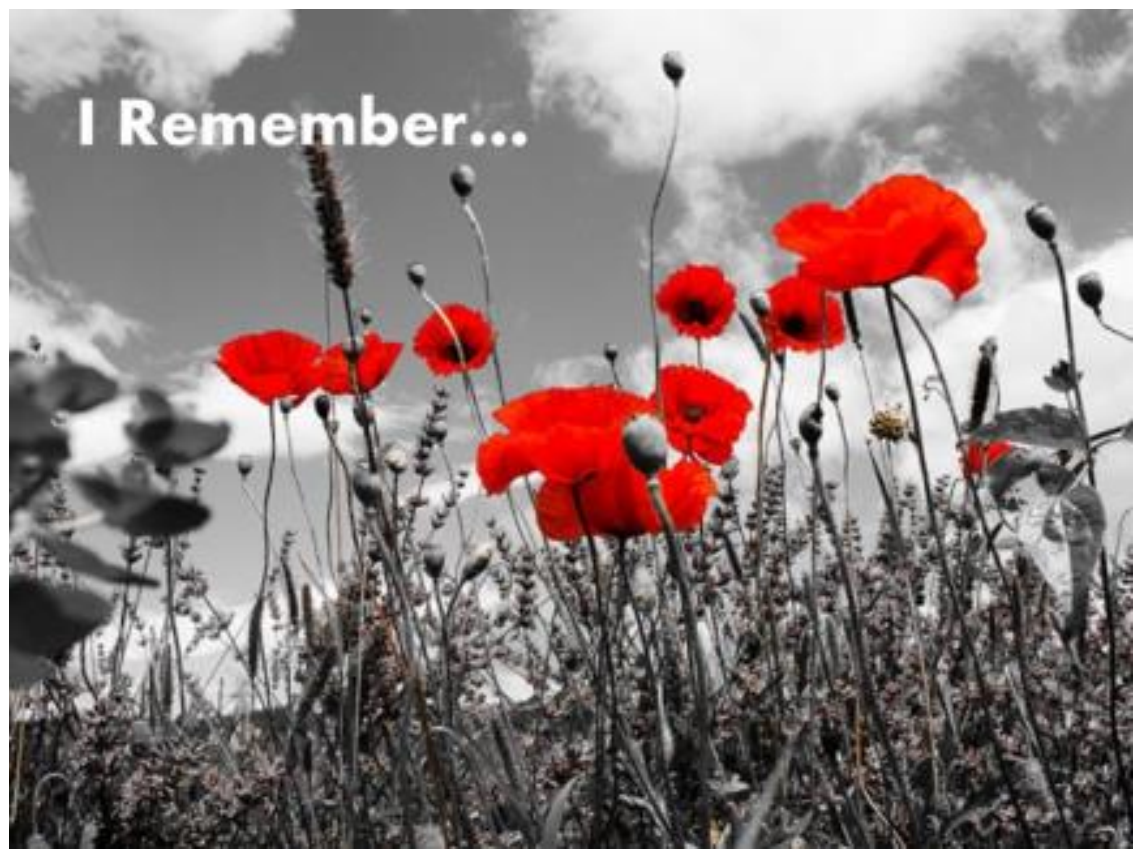

Verbal Appeal (Independent Self-Construal): Please donate to support your veterans. You can honour those who have given so much, and help by donating. Show that you remember.

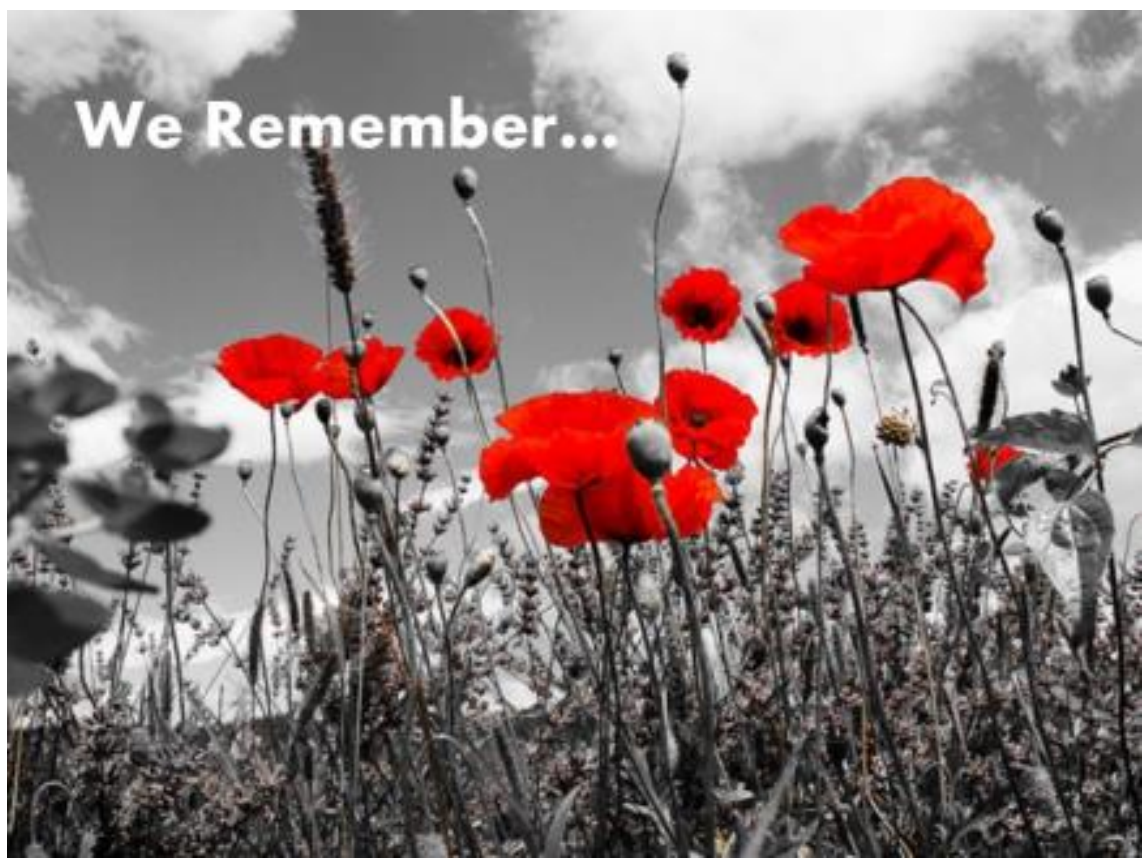

Verbal Appeal (Interdependent Self-Construal): Please donate to support our veterans. We can honour those who have given so much, and help by donating. Show that we remember. 
APPENDIX B - STIMULI USED IN STUDIES 2, 4A AND 5

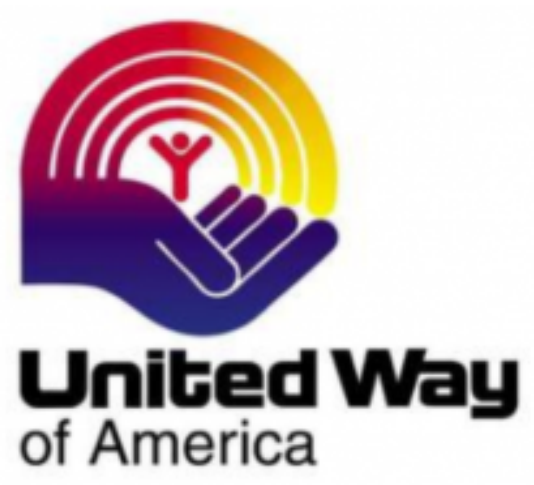

The United Way of America is national system of volunteers, contributors, and local charities helping people in their own communities.

In communities across the United States the United Way is working every day to ensure every child has a quality education, every family has a stable income, and all Americans enjoy good health. But to continue their vital work, they need support, and every little bit helps.

By donating to the United Way:

- You'll help those less fortunate.

- You'll help your community become a better place for everyone. 


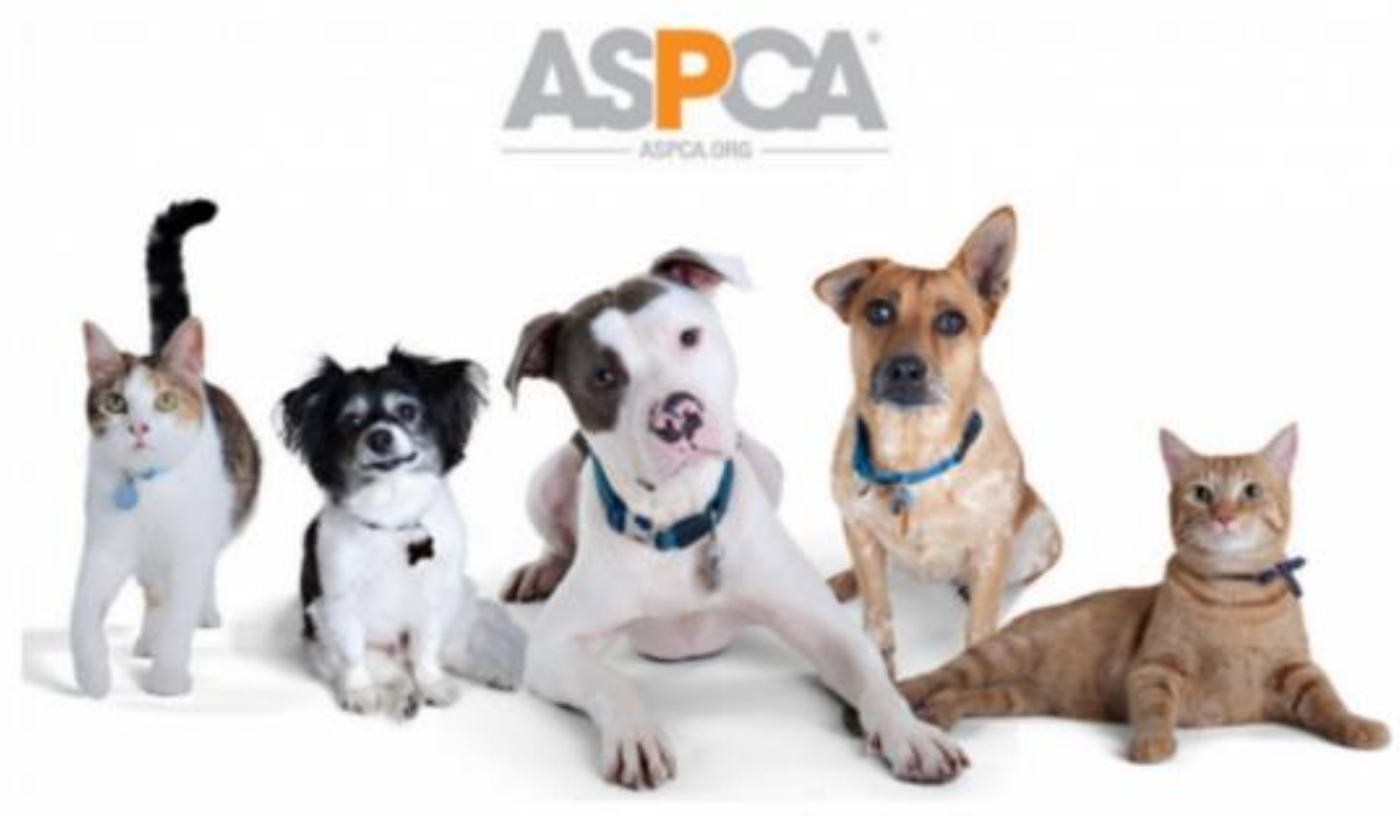

Every year, tens of thousands of animals are rescued, sheltered, placed in homes, or reunited with their owners as a result of the work of ASPCA staff and volunteers. The ASPCA is a non-profit organization funded primarily by public donations. Funds are used to prevent cruelty and to promote the welfare of animals through a wide range of services including community and education programs, and the operation of Pet Registries nation-wide.

To continue their work, they need support, and every little bit helps.

By supporting the ASPCA, you can help. 
APPENDIX D - STIMULI USED IN STUDIES 4B AND 6

\section{BUILDING A GRADUATION NATION to Education}

Every year, thousands of students make the life-altering decision to drop out of high school. In low-income communities across the nation, the high school dropout rate can reach up to 50 percent, taking a dramatic toll on communities, health and justice systems, and the economy. Pathways addresses barriers to education by providing leadership, expertise, and a community-based program proven to lower dropout rates.

To continue their work, they need support, and every little bit helps.

By supporting the fund, you can help. 


\section{REFERENCES}

Abele, Andrea E. and Bogdan Wojciszke (2007), “Agency and Communion from the Perspective of Self Versus Others," Journal of Personality and Social Psychology, 93 (5), 751-63.

Andreoni, James and Ragan Petrie (2004), "Public Goods Experiments Without Confidentiality:

A Glimpse into Fund-Raising," Journal of Public Economics, 88 (7/8), 1605-23.

Ariely, Dan, Anat Bracha, and Stephan Meier (2009), "Doing Good or Doing Well? Image Motivation and Monetary Incentives in Behaving Prosocially," The American Economic Review, 99 (1), 544-55.

Argo, Jennifer J., Katherine White, and Darren. W. Dahl (2006), "Social Comparison Theory and Deception in the Interpersonal Exchange of Consumption Information," Journal of Consumer Research, 33 (1), 99-108.

Bandura, Albert (1989), "Human Agency in Social Cognitive Theory," American Psychologist, 44 (9), 1175-84.

Basil, Debra Z., Mary S. Runte, M. Easwaramoorthy, and Cathy Barr (2009), “Company Support for Employee Volunteering: A national Survey of Companies in Canada," Journal of Business Ethics, 85 (2), 387-98.

Benabou, Roland and Jean Tirole (2006), "Incentives and Prosocial Behavior," American Economic Review, 96 (5), 1652-78.

Bendapudi, Neeli, Surendra N. Singh, and Venkat Bendapudi (1996), "Enhancing Helping Behavior: An Integrative Framework for Promotion Planning,” Journal of Marketing, 60 (3), 33-49.

Brewer, Marilynn B. and Wendi Gardner (1996), "Who Is This 'We'? Levels of Collective Identity and Self-Representations," Journal of Personality and Social Psychology, 71 (1), $83-93$. 
Bullock, John G., Donald P. Green, and Shang E. Ha (2010), "Yes, But What's the Mechanism? (Don't Expect an Easy Answer)," Journal of Personality and Social Psychology, 98 (April), 550-8.

Burton, Alex K., Jonathan S. Gore, and Jennifer Sturgeon (2012), "The Role of Relational SelfConstrual in Reactions to Charity Advertisements," Self and Identity, 11 (3), 343-59.

Chen, Beiwen, Maarten Vansteenkiste, Wim Beyers, Liesbet Boone, Edward L. Deci, Jolene Van der Kaap-Deeder, Bart Duriez, Willy Lens, Lennia Matos, Athanasios Mouratidis, Richard M. Ryan, Kennon M. Sheldon, Bart Soenens, Stijn Van Petegem, and Joke Verstuyf (2015), "Basic Psychological Need Satisfaction, Need Frustration, and Need Strength Across Four Cultures," Motivation and Emotion, 39 (2), 216-36.

Children's Miracle Network (2016), "Walmart Campaign in Full Swing," https://mclanechildrens.childrensmiraclenetworkhospitals.org/walmart-campaign-fullswing/.

Conway, Lucian Gideon III, Andrew G. Ryder, Roger G. Tweed, Bryan W. Sokol (2001), "International Cultural Variation: Exploring Further Implications of Collectivism Within the United States," Journal of Cross-Cultural Pscyhology, 32 (6), 681-97.

Cutright, Keisha M. and Adriana Samper (2014), “Doing It the Hard Way: How Low Control Drives Preferences for High-Effort Products and Services," Journal of Consumer Research, 41 (3), 730-45.

Deci, Edward L. and Richard M. Ryan (1985), Intrinsic Motivation and Self-Determination in Human Behavior, New York: Springer.

Duclos, Rod and Alixandra Barasch (2014), "Prosocial Behavior in Intergroup Relations: How Donor Self-Construal and Recipient Group-Membership Shape Generosity," Journal of Consumer Research, 41 (1), 93-108.

Fisher, Robert J. and David Ackerman (1998), "The Effects of Recognition and Group Need on Volunteerism: A Social Norm Perspective," Journal of Consumer Research, 25 (3), 262 75. 
Froming, William J., G. Rex Walker, and Kevin J. Lopyan (1982), "Public and Private SelfAwareness: When Personal Attitudes Conflict with Societal Expectations," Journal of Experimental Social Psychology, 18 (5), 476-87.

Gardner, Wendi L., Shira Gabriel, and Angela Y. Lee (1999), "I" Value Freedom But "We" Value Relationships: Self-Construal Priming Mirrors Cultural Differences in Judgment," Psychological Science, 10, 321-26.

Giving USA Foundation (2016), "See the Numbers: Giving USA 2016 Infographic," https://givingusa.org/see-the-numbers-giving-usa-2016-infographic/

Grant, Adam M. and David M. Mayer (2009), “Good Soldiers and Good Actors: Prosocial and Impression Management Motives as Interactive Predictors of Affiliative Citizenship Behaviors," Journal of Applied Psychology, 94, 900-12.

Han, DaHee, Ashok K. Lalwani, and Adam Duhachek (2017), "Power Distance Belief, Power, and Charitable Giving," Journal of Consumer Research, 44, 182-95.

Harbaugh, William T. (1998), “The Prestige Motive for Making Charitable Transfers,” American Economic Review, 88, 277-82.

Hayes, Andrew F. (2013), An Introduction to Mediation, Moderation, and Conditional Process Analysis: A Regression-Based Approach, New York, NY: Guilford.

Hofstede, Geert (2001), Culture's Consequences: Comparing Values, Behaviors, Institutions, and Organizations Across Nations, $2^{\text {nd }}$ ed., Thousand Oaks, CA: Sage.

Holmes, John G., Dale T. Miller, and Melvin J. Lerner (2002), “Committing Altruism under the Cloak of Self-Interest: The Exchange Fiction," Journal of Experimental Social Psychology, 38 (2), 144-51.

Ji, Lijun, Norbert Schwarz, and Richard E. Nisbett (2000), “Culture, Autobiographical Memory, and Behavioral Frequency Reports: Measurement Issues in Cross-Cultural Studies," Personality and Social Psychology Bulletin, 26 (5), 586-94.

Karlan, Dean and Margaret A. McConnell (2014), "Hey Look at Me: The Effect of Giving Circles on Giving," Journal of Economic Behavior \& Organization, 106 (C), 402-12. 
Kemmelmeier, Markus, Edina E. Jambor, and Joyce Letner (2006), “Individualism and Good Works: Cultural Variation in Giving and Volunteering Across the United States," Journal of Cross-Cultural Pscyhology, 37 (3), 327-44.

Kotler, Philip and Nancy Lee (2005), Corporate Social Responsibility: Doing the Most Good for Your Company and Your Cause, New York, NY: Wiley.

Kristofferson, Kirk, Katherine White, and John Peloza (2014), “The Nature of Slacktivism: How the Social Observability of an Initial Act of Token Support Affects Subsequent Prosocial Action," Journal of Consumer Research, 40 (6), 1149-66.

Kulow, Katina and Thomas Kramer (2016), "In Pursuit of Good Karma: When Charitable Appeals to Do Right Go Wrong," Journal of Consumer Research, 43 (2), 334-53.

Lacetera, Nicola and Mario Macis (2010), "Social Image Concerns and Prosocial Behavior: Field Evidence from a Nonlinear Incentive Scheme," Journal of Economic Behavior \& Organization, 76(2), 225-237.

Lalwani, Ashok K. and Sharon Shavitt (2009), “The 'Me' I Claim to Be: Cultural Self-Construal Elicits Self-Presentational Goal Pursuit," Journal of Personality and Social Psychology, 97 (1), 88-102.

Lee, Saerom, Karen Page Winterich, and William T. Ross Jr. (2014), 'I’m Moral, but I Won’t Help You: The Distinct Roles of Empathy and Justice in Donations," Journal of Consumer Research, 41(3), 678-96.

Lerner, Jennifer S. and Philip E. Tetlock (1999), "Accounting for the Effects of Accountability," Psychological Bulletin, 125, 255-75.

MacDonnell, Rhiannon and Katherine White (2015), "How Construals of Money Versus Time Impact Consumer Charitable Giving," Journal of Consumer Research, 42 (4), 551-63.

Markus, Hazel Rose and Shinobu Kitayama (1991), "Culture and the Self: Implications for Cognition, Emotion, and Motivation," Psychological Review, 98 (2), 244-53.

Miller, Dale T. (1999), “The Norm of Self-Interest,” American Psychologist, 54 (12), 1053-60. 
Moorman, Robert H. and Gerald L. Blakely (1995), "Individualism-Collectivism as an Individual Difference Predictor of Organizational Citizenship Behavior," Journal of Organizational Behavior, 16, 127-42.

Nelson, Michelle, Frederic Brunel, Magne Supphellen, and Rajesh Manchanda (2006), "Effects of Culture, Gender, and Moral Obligations on Responses to Charity Advertising Across Masculine and Feminine Cultures," Journal of Consumer Psychology, 16 (1), 45-56.

Park, Kiwan and Seojin Stacey Lee (2015), “The Role of Beneficiaries' Group Identity in Determining Successful Appeal Strategies for Charitable Giving,” Psychology \& Marketing, 32 (12), 1117-32.

Ratner, Rebecca and Dale T. Miller, (1998) "The Norm of Self-Interest and Its Effects on Social Action," Journal of Personality and Social Psychology, 81, 5-16.

Ratner, Rebecca and Kahn, Barbara (2002), “The Impact of Private versus Public Consumption on Variety-Seeking Behavior,” Journal of Consumer Research, 29 (2), 246-57.

Ryan, Richard M. and Edward L. Deci (2000), "Self-determination Theory and the Facilitation of Intrinsic Motivation, Social Development, and Well-being," American Psychologist, $55(1), 68-78$.

Ryan, Richard M., Edward L. Deci, and Wendy S. Grolnick (1995), “Autonomy, Relatedness, and the Self: Their Relation to Development and Psychopathology," in Developmental Psychopathology, Vol. 1, Ed. Dante Cicchetti and Donald J. Cohen, New York: Wiley, 618-55.

Schlosser, Ann E. and Eric Levy (2016), "Helping Others or Oneself: How Direction of Comparison Affects Prosocial Behavior,” Journal of Consumer Psychology, 26 (4), 46173.

Seo, Joon Yong and Debra L. Scammon (2014), "Does Feeling Holier Than Others Predict Good Deeds? Self-construal, Self-enhancement and Helping Behavior," Journal of Consumer Marketing, 31 (6/7), 441-51.

Singelis, Theodore M. (1994), “The Measurement of Independent and Interdependent Self- 
Construals," Personality and Social Psychology Bulletin, 20 (October), 580-91.

Spencer, Steven J., Mark P. Zanna, and Geoffrey T. Fong (2005), "Establishing a Causal Chain: Why Experiments are Often More Effective than Mediational Analyses in Examining Psychological Processes," Journal of Personality and Social Psychology, 89 (December), $845-51$.

Stojcic, Ivana, Lu Kewen, and Ren Xiaopeng (2016), “Does Uncertainty Avoidance Keep Charity Away? Comparative Research Between Charitable Behavior and 79 National Cultures," Culture and Brain, 4 (1), 1-20.

Turner, Rhiannon N. and Richard J. Crisp (2007), “Essential Social Psychology,” London, UK: Sage.

Wang, Xia and Luqiong Tong (2015), "Hide the Light or Let it Shine? Examining the Factors Influencing the Effect of Publicizing Donations on Donors' Happiness," International Journal of Research in Marketing, 32, 418-24.

White, Katherine, Bonnie Simpson, and Jennifer J. Argo (2014), “The Motivating Role of Dissociative Out-Groups in Encouraging Positive Consumer Behaviors," Journal of Marketing Research, 51 (August), 433-47.

White, Katherine and Bonnie Simpson (2013), “When Do (and Don't) Normative Appeals Influence Sustainable Consumer Behaviors?” Journal of Marketing, 77 (March), 78-95.

White, Katherine, Jennifer J. Argo, and Jaideep Sengupta (2012), "Dissociative versus Associative Responses to Social Identity Threat: The Role of Consumer Self-Construal," Journal of Consumer Research, 39 (4), 704-19.

White, Katherine and Jennifer J. Argo (2011), “When Imitation Doesn't Flatter: The Role of Consumer Distinctiveness in Response to Mimicry,” Journal of Consumer Research, 38 (4), 667-680.

White, Katherine and John Peloza (2009), "Self-Benefit Versus Other-Benefit Marketing Appeals: Their Effectiveness in Generating Charitable Support," Journal of Marketing, 73 (4), 109-24. 
Wien, Anders Hauge and Svein Ottar Olsen (2014), "Understanding the Relationship between Individualism and Word of Mouth: A Self-Enhancement Explanation,” Psychology \& Marketing, 31 (6), 416-25.

Winer, Ben J. (ed.) (1971), “Statistical Principles in Experimental Design,” New York, NY: McGraw Hill.

Winterich, Karen Page and Yinlong Zhang (2014), “Accepting Inequality Deters Responsibility: How Power Distance Decreases Charitable Behavior,” Journal of Consumer Research, $41(2), 274-93$.

Winterich, Karen Page, Karl Aquino, Vikas Mittal, and Richard Swartz (2013), "When Moral Identity Symbolization Motivates Prosocial Behavior: The Role of Recognition and Moral Identity Internalization,” Journal of Applied Psychology, 98 (5), 759-70.

Winterich, Karen Page and Michael J. Barone (2011), "Warm Glow or Cold, Hard Cash? Social Identity Effects on Consumer Choice for Donation Versus Discount Promotions," Journal of Marketing Research, 48 (5), 855-68.

Yoo, Boonghee, Naveen Donthu, and Tomasz Lenartowicz (2011), “Measuring Hofstede's Five Dimensions of Cultural Values at the Individual Level: Development and Validation of CVSCALE," Journal of International Consumer Marketing, 23 (3/4), 193-210. 
FIGURE 1A

STUDY 1 RESULTS

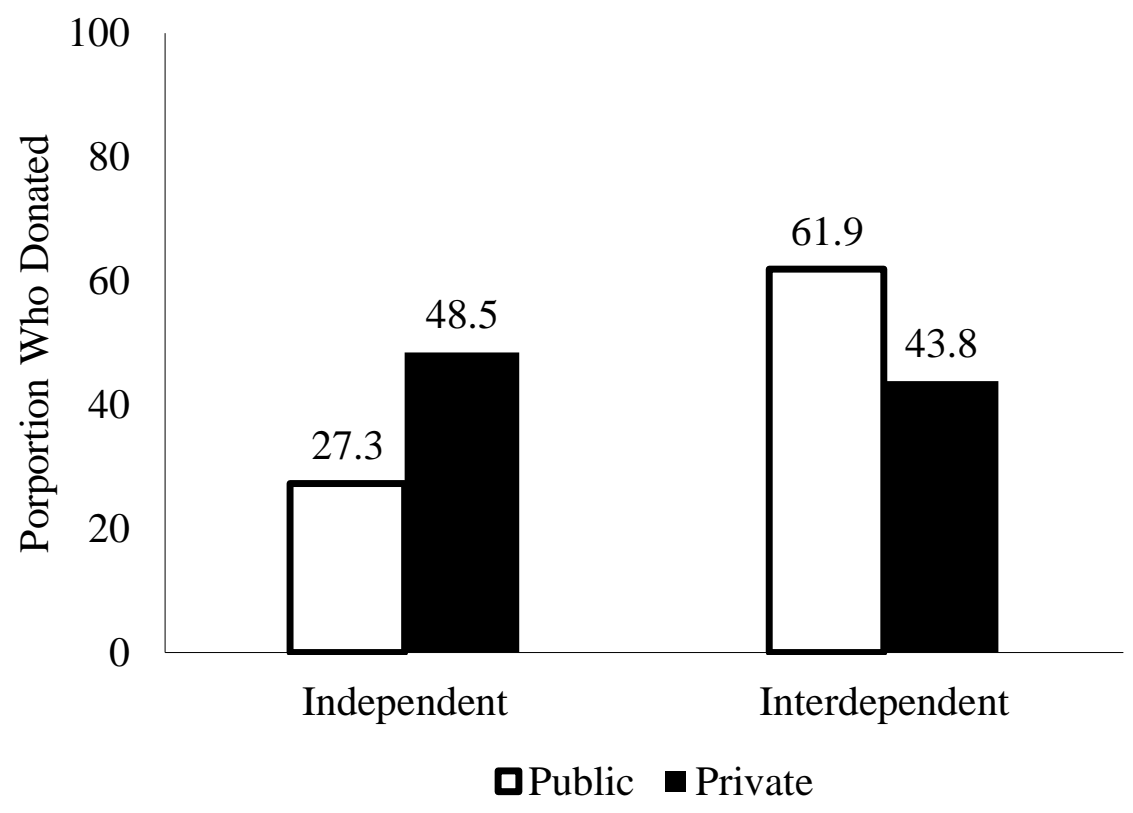

FIGURE 1B

STUDY 1 RESULTS

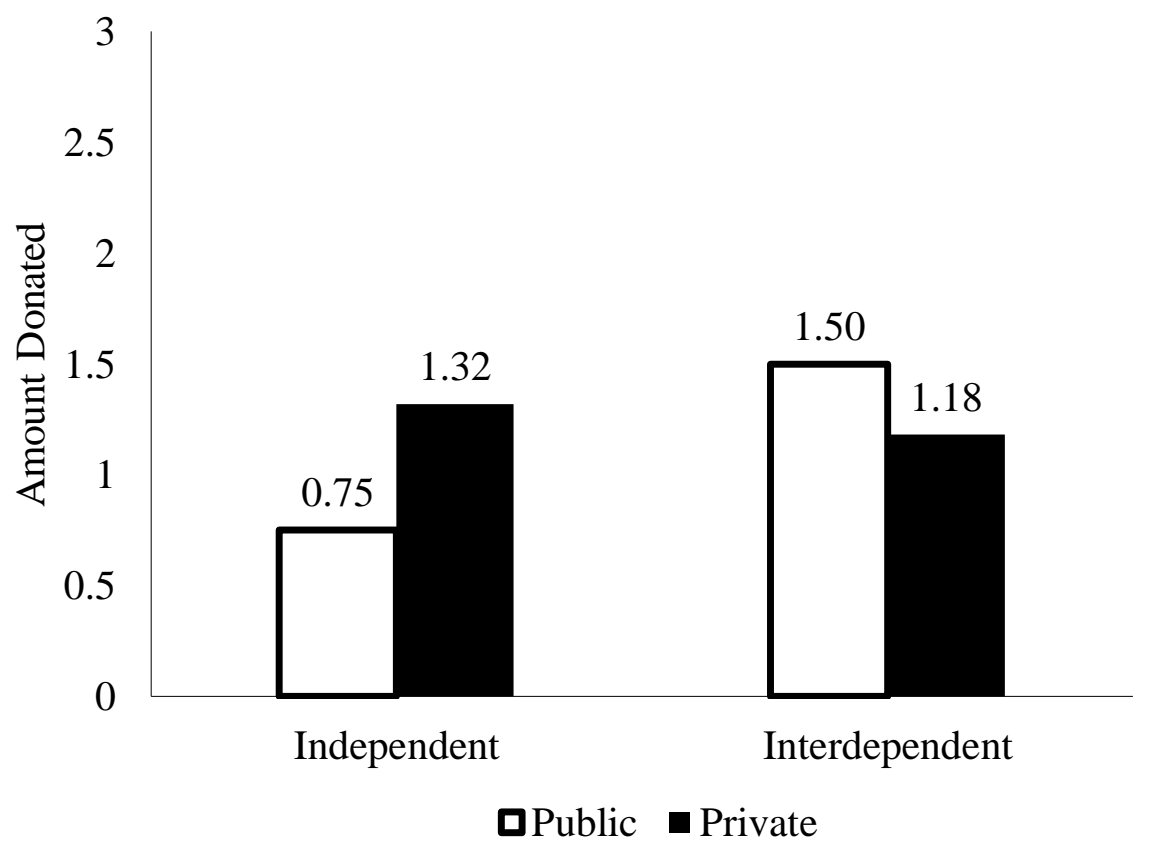


FIGURE 2

STUDY 2 RESULTS

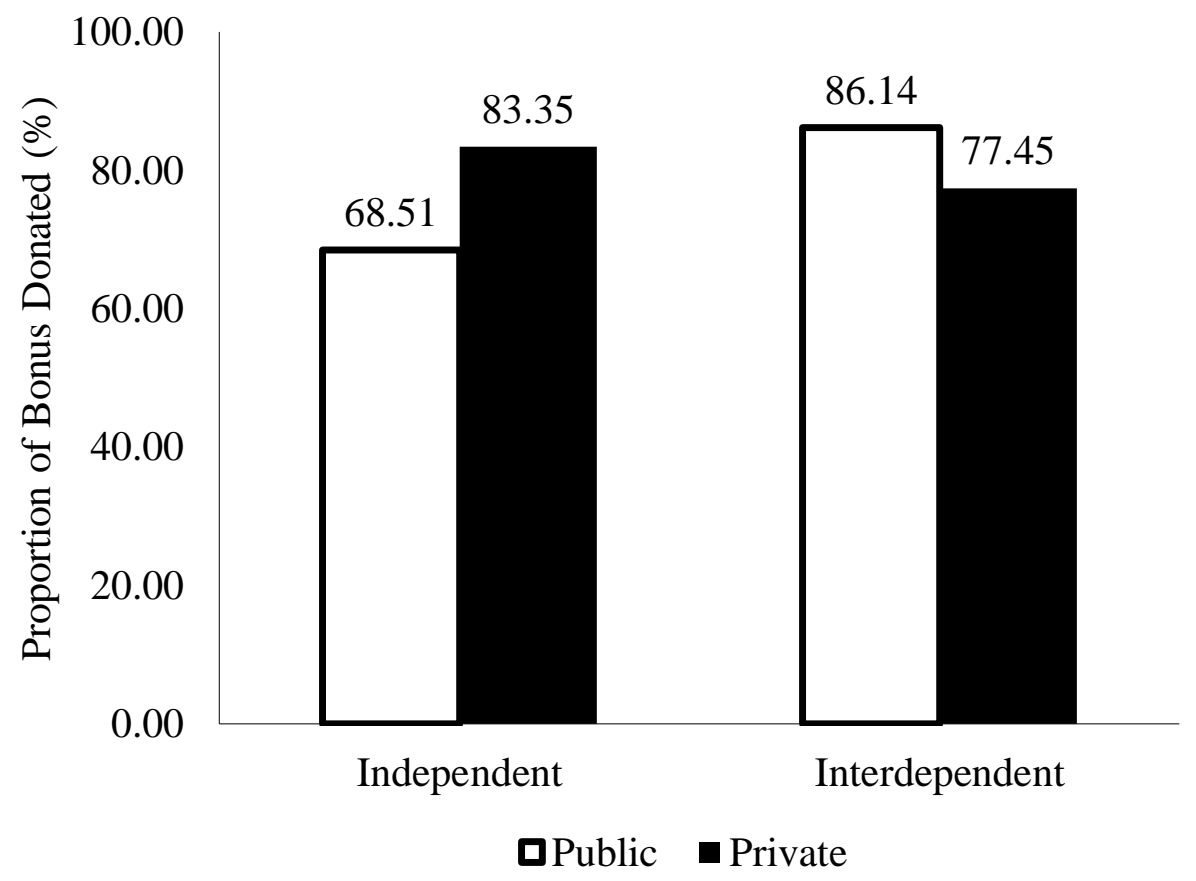


FIGURE 3

STUDY 3 RESULTS

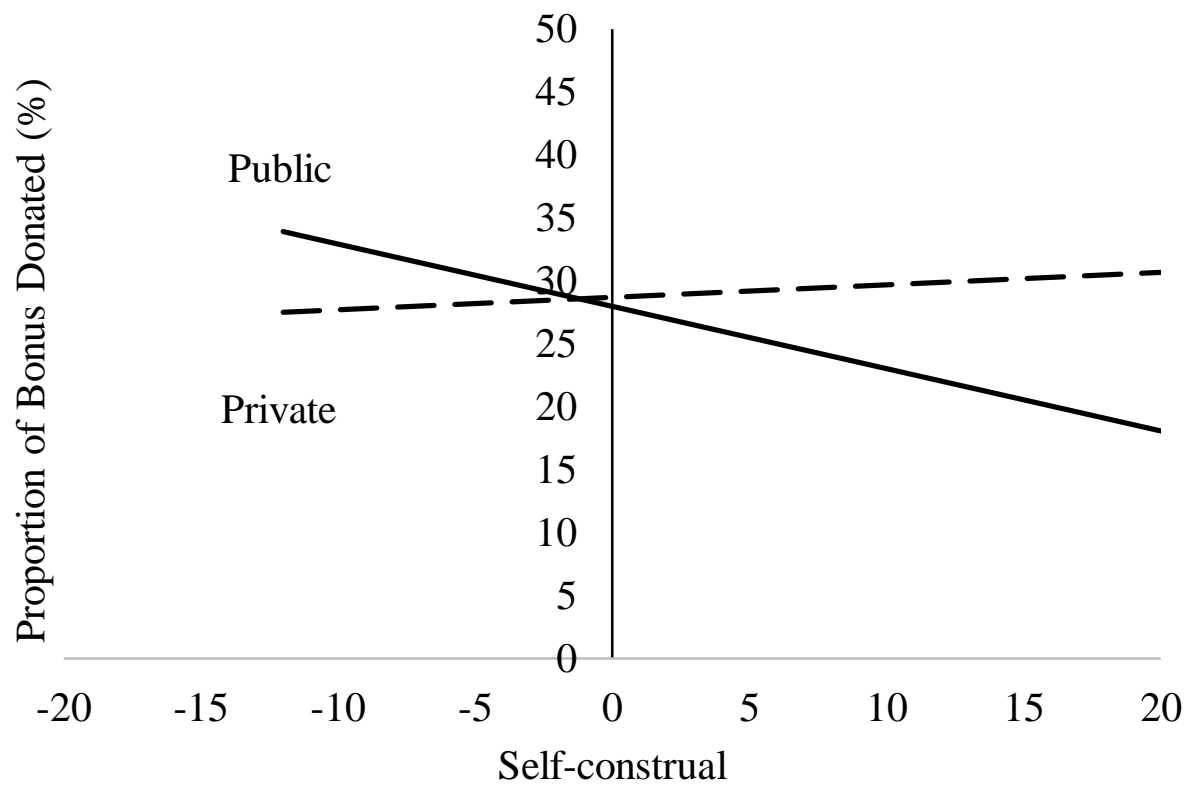

(higher values indicate a more independent self-construal) 


\section{FIGURE 4A}

STUDY 4A RESULTS

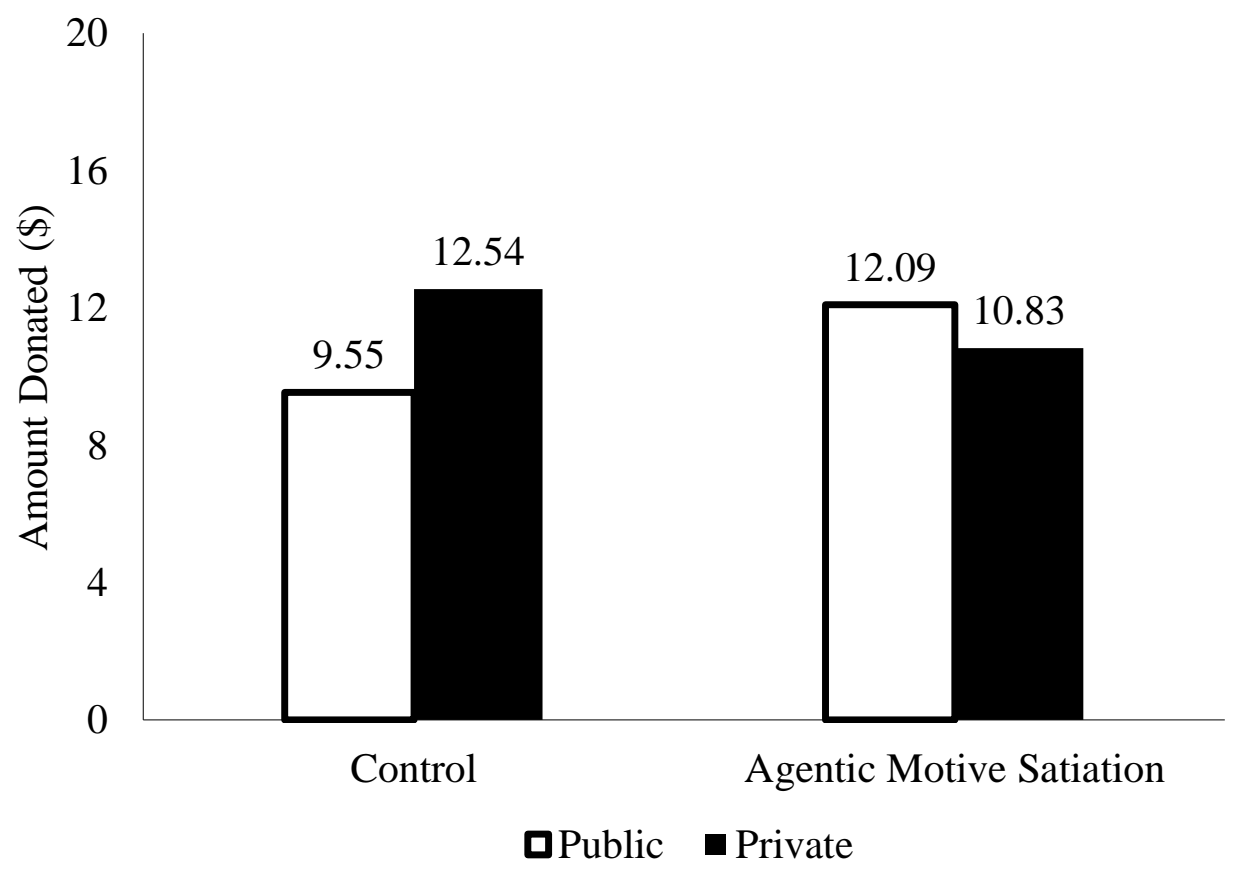

FIGURE 4B

STUDY 4B RESULTS

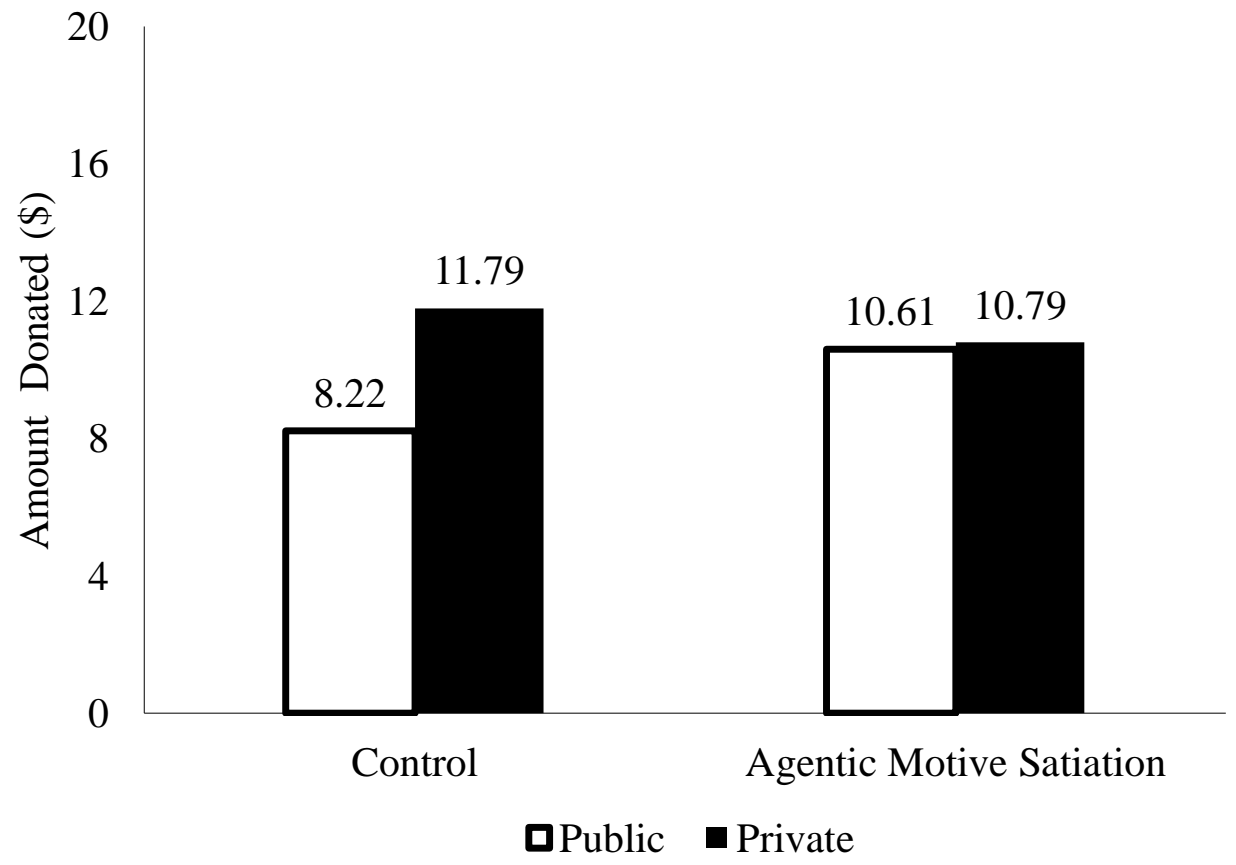


FIGURE 5

STUDY 5 RESULTS

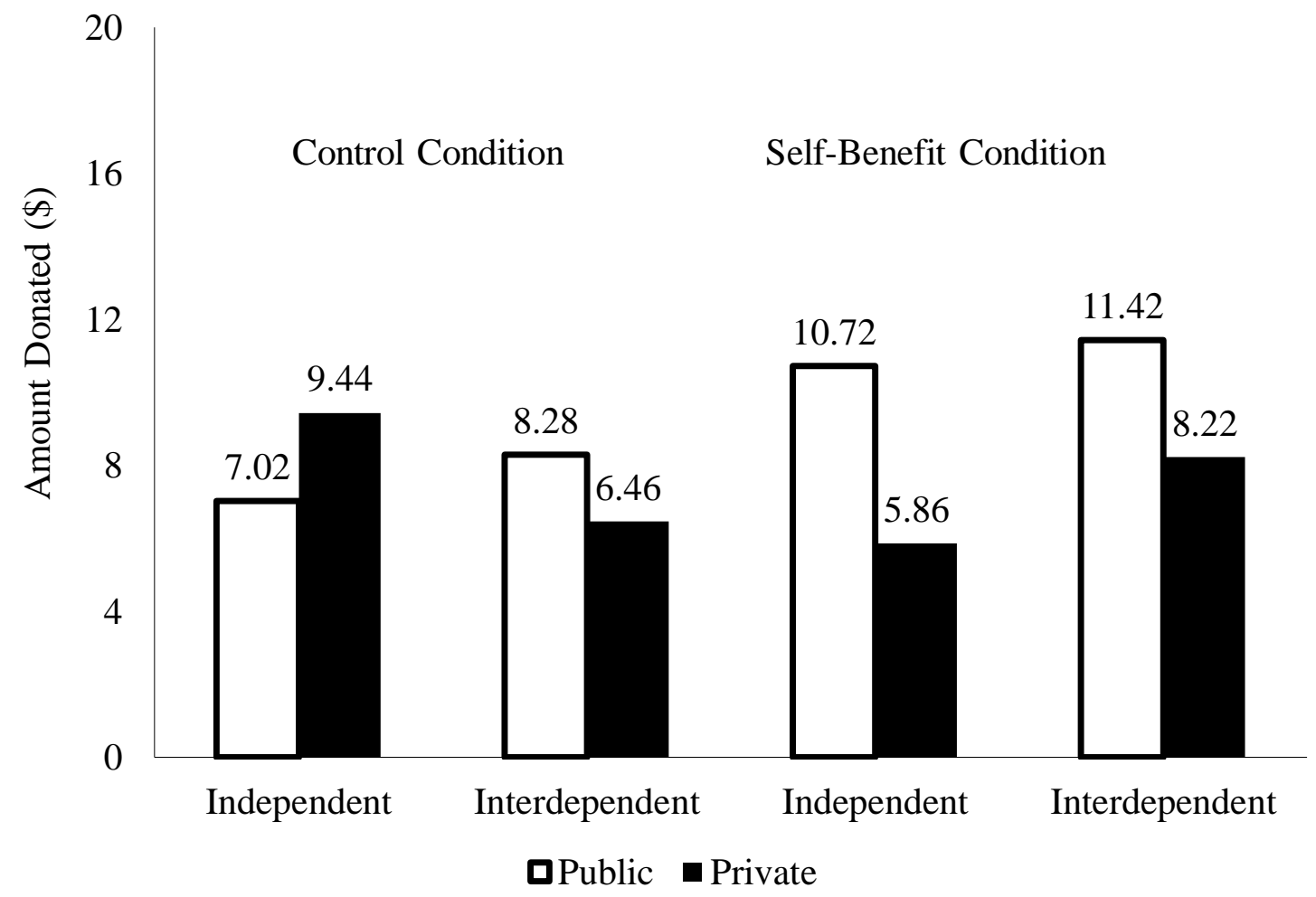


FIGURE 6

STUDY 6 RESULTS

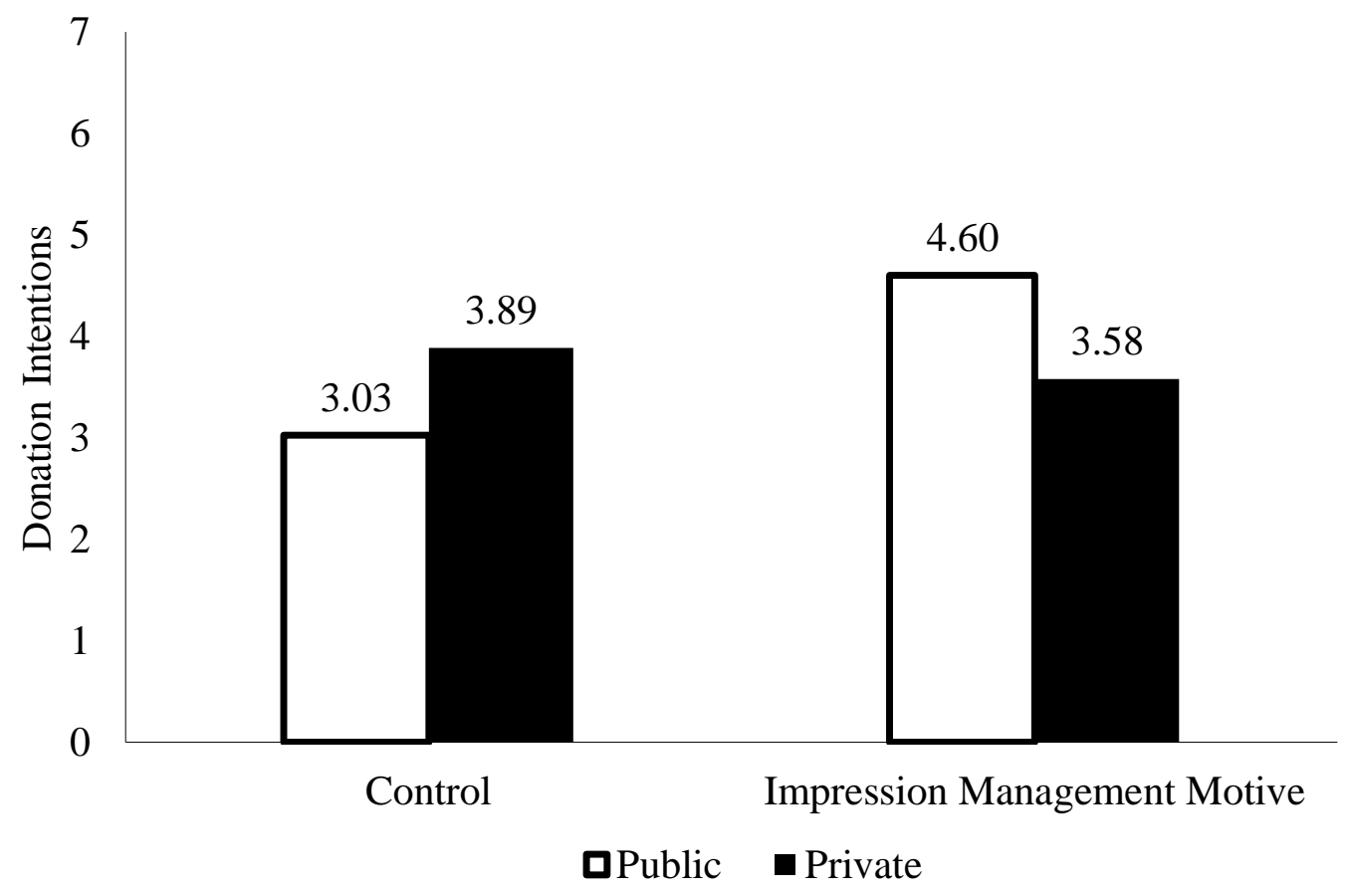


Headings List

1) PUBLIC RECOGNITION AND CHARITABLE GIVING

2) The Role of Self-Construal

2) Independent Self-Construal, Agency, and Public Charitable Giving

1) OVERVIEW OF THE CURRENT INVESTIGATION

1) STUDY 1

2) Method

3) Participants and Design

3) Procedure.

2) Results and Discussion

3) Willingness to Donate.

3) Donation Amount.

1) STUDY 2

2) Method

3) Participants and Design.

3) Procedure.

2) Results and Discussion

1) STUDY 3

2) Method

3) Participants and Design.

3) Procedure.

2) Results and Discussion

3) Manipulation Checks. 
3) Donations.

3) Mediation by Agency.

3) Additional Measures.

1) STUDY 4A

2) Method

3) Participants and Design.

3) Procedure.

2) Results

1) STUDY 4B

2) Method

3) Participants and Design.

3) Procedure.

2) Results and Discussion

3) Donations.

3) Mediation by Agency.

1) STUDY 5

2) Method

3) Participants and Design.

3) Procedure.

2) Results and Discussion

1) STUDY 6

2) Method

3) Participants and Design. 
3) Procedure.

2) Results and Discussion

1) GENERAL DISCUSSION

2) Theoretical Contribution

2) Practical Implications and Directions for Future Research 